Corrigendum

\title{
Association between vitamin B12 intake and EURRECA's prioritized biomarkers of vitamin B12 in young populations: a systematic review - CORRIGENDUM
}

Iris Iglesia, Rosalie AM Dhonukshe-Rutten, Silvia Bel-Serrat, Esme’e L Doets, Adrienne EJM Cavelaars, Pieter van 't Veer, Mariela Nissenshohn, Vassiliki Benetou, Marı'a Hermoso, Cristiana Berti, Lisette CPGM de Groot and Luis A Moreno

First published online 4 December 2014

doi: 10.1017/S1368980012003953 Published by Cambridge University Press, 13 September 2012.

Author Mariela Nissensohn's name was originally misspelled in this paper. The authors apologise for this error.

\section{Reference}

Iglesia I, Dhonukshe-Rutten RA, Bel-Serrat S, Doets EL, Cavelaars AE, van 't Veer P, Nissensohn M, Benetou V, Hermoso M, Berti C, de Groot LC \& Moreno L (2013) Association between vitamin B12 intake and EURRECA's prioritized biomarkers of vitamin B12 in young populations: a systematic review. Public Health Nutr. 16, 1843-60. doi: 10.1017/S1368980012003953. 